GSA Data Repository 2018195

\title{
Marine redox fluctuation as a potential trigger for the Cambrian explosion
}

\author{
Guang-Yi Wei ${ }^{1,2^{*}}$, Noah J. Planavsky ${ }^{2}$, Lidya G. Tarhan ${ }^{2}$, Xi Chen ${ }^{1}$, Wei Wei ${ }^{1}$, Da Li $^{1^{*}}$, Hong-Fei Ling ${ }^{1 *}$
}

${ }^{1}$ State Key Laboratory for Mineral Deposits Research, Department of Earth Sciences, School of Earth Sciences and Engineering, Nanjing University, 163 Xianlin Avenue, Nanjing 210023, China.

${ }^{2}$ Department of Geology and Geophysics, Yale University, New Haven, CT 06520, USA

*Corresponding author

E-mail: wgynjues@gmail.com, guangyi.wei@yale.edu (G.-Y.Wei); 1ida@nju.edu.cn (D. Li);

hfling@,nju.edu.cn (H.-F. Ling).

\section{DR1. Sample digestion and $U$ isotope analyses}

Approximately $250 \mathrm{mg}$ of individual carbonate samples were drilled into 200 mesh powder, then leached with $1 \mathrm{M}$ hydrochloric acid $(\mathrm{HCl})$. The leachate was used to analyze major and trace elements and $U$ isotopes. Major and trace elements were measured on a Thermo Scientific Finnigan Element XR ICP-MS at the Yale Metal Geochemistry Center (YMGC). External reproducibility was better than $5 \%$ based on long-term analyses of the OSIL seawater standard. U isotopes $\left(\delta^{238} U\right)$ were measured on a Thermo Scientific Finnigan Neptune plus MCICP-MS at YMGC, using a UTEVA resin method for purifying uranium and a ${ }^{233} \mathrm{U}_{-}^{236} \mathrm{U}$ double spike for fractionation calibration.

A ${ }^{233} \mathrm{U}^{236} \mathrm{U}$ double spike method was used to correct for instrumental mass bias of MCICP-MS and potential isotopic fractionation during uranium separation. The sample and double spike mixing solution which yields a ${ }^{238} \mathrm{U} /{ }^{236} \mathrm{U}$ ratio of $\sim 30$, was dried at 98 , then re-dissolved with $3 \mathrm{~N} \mathrm{HNO}_{3}$. Uranium in the sample was separated with the UTEVA ion exchange resin (based on Weyer et al., 2008). The UTEVA resin (1.0 ml for each column) was cleaned with $0.05 \mathrm{~N} \mathrm{HCl}$ and MQ2 water, then conditioned with $3 \mathrm{~N} \mathrm{HNO}_{3}$. Samples were dissolved in $3 \mathrm{~N}$ $\mathrm{HNO}_{3}$, then loaded on to the UTEVA resin. $3 \mathrm{~N} \mathrm{HNO}_{3}$ was used to remove most matrix elements from the column, leaving a fraction of Th and $U$ in the column. After removal of matrix material, $10 \mathrm{~N} \mathrm{HCl}$ and $5 \mathrm{~N} \mathrm{HCl}$ were used to remove Th from the column, then $\mathrm{U}$ was eluted with $0.05 \mathrm{~N}$ $\mathrm{HCl}$. The $\mathrm{U}$ fraction was dried at the temperature of 98 and re-dissolved with $1 \mathrm{ml} 5 \% \mathrm{HNO}_{3}$ for analysis. 
U isotopes were measured with Thermo Neptune plus MC-ICP-MC instruments with a ESI Apex IR desolvating system. U isotope analyses were under low-resolution (LR) and beam intensities for ${ }^{232} \mathrm{Th},{ }^{233} \mathrm{U},{ }^{234} \mathrm{U},{ }^{235} \mathrm{U},{ }^{236} \mathrm{U},{ }^{238} \mathrm{U}$ were measured in Faraday cups of L3, L2, L1, C, $\mathrm{H} 1, \mathrm{H} 3$, respectively. ${ }^{232} \mathrm{Th}$ was analysed in order to monitor the interference of ${ }^{234} \mathrm{Th}$ in the sample solution. All of the $\mathrm{U}$ isotope ion beam signals were measured with the $10^{11} \Omega$ resistance. Each sample was measured with 4 blocks composed of 10 cycles (4.194 s integration time for every cycle). The CRM 112a standard with addition of double spike was analyzed every 3 samples to monitor drift in instrumental mass bias. ${ }^{238} \mathrm{U} /{ }^{235} \mathrm{U}$ values are presented as $\delta^{238} \mathrm{U}$ :

$$
\delta^{238} \mathrm{U}=\left[\left({ }^{238} \mathrm{U} /{ }^{235} \mathrm{U}\right)_{\text {sample }} /\left({ }^{238} \mathrm{U} /{ }^{235} \mathrm{U}\right)_{\text {CRM 112a }}-1\right] \times 1000
$$

$\delta^{238} \mathrm{U}$ and ${ }^{234} \mathrm{U} /{ }^{238} \mathrm{U}$ values of each sample were normalized to the average values of the bracketing CRM 112a, assuming $\delta^{238} \mathrm{U}_{\mathrm{CRM} 112 \mathrm{a}}=0 \%$ and $\left({ }^{234} \mathrm{U} /{ }^{238} \mathrm{U}\right)_{\mathrm{CRM} 112 \mathrm{a}}=0.9619 .{ }^{234} \mathrm{U} /{ }^{238} \mathrm{U}$ values are calculated using decay constants for ${ }^{238} \mathrm{U}$ and ${ }^{234} \mathrm{U}$ of $1.55 \times 10^{-10}$ and $2.83 \times 10^{-6}$, respectively (Cheng et al., 2013; Villa et al., 2016). Measurement uncertainty for each sample is better than $0.04 \%$ (2SE) and external reproducibility of $U$ isotope analyses was, on average, $0.06 \%$ (2SD) based on duplicate measurements of the NOD-A-1 USGS standard ( $\mathrm{n}=10)$ and CRM 112 $\mathrm{U}$ isotope standard $(\mathrm{n}=50)$.

\section{DR2. Ce anomaly calculation}

$\mathrm{Ce}$ anomaly is calculated using equation $\mathrm{Ce} / \mathrm{Ce} \mathrm{C}^{*}=\mathrm{Ce}_{\mathrm{N}} /\left(\operatorname{Pr}_{\mathrm{N}}{ }^{2} / \mathrm{Nd}_{\mathrm{N}}\right)$ of Lawrence et al. (2006) to avoid the effect of La overabundance that may disguise the genuine Ce anomaly (Ling et al., 2013), where lower subscript $\mathrm{N}$ denotes normalization of concentrations against the postArchean Australian shale (PAAS; McLennan, 1989).

\section{DR3. Geological background}

\subsection{Yangtze Gorges region}

The Ediacaran and the early Cambrian successions in this region consist of the Doushantuo Formation, Dengying Formation (the Gaojiaxi section in this study) and Yanjiahe Formation (the Yanjiahe section in this study), which records sedimentary deposition in shallow water below or near wave base in a continental inner shelf. According to lithostratigraphic criteria, the Doushantuo Formation in the Yangtze Gorges area can be subdivided into four members (e.g., Jiang et al., 2011). Doushantuo Member I comprises a ca. 5-m-thick cap dolostone overlying the Marinoan glacial diamictite of the Nantuo Formation. The bottom part of the cap dolostone contains a volcanic ash layer that has been dated at $635.2 \pm 0.6 \mathrm{Ma}$ with zircon 
U-Pb methods (Condon et al., 2005). Doushantuo Member II is ca. $70 \mathrm{~m}$ thick and comprises interbedded organic-rich shale and dolostone beds with abundant pea-sized cherty nodules which contain complex microfossils including acanthomorphic acritarchs, probable animal eggs, embryos, multicellular algae, and cyanobacteria (e.g., Yin et al., 2007; McFadden et al., 2008). Doushantuo Member III is a ca. 50-m-thick interval comprised of dolostones intercalated with cherty layers in the lower part and interbedded with limestone beds in the upper part. Doushantuo Member IV is characterized by a 10-m-thick organic-rich black shale interval used as marker beds for stratigraphic correlation (e.g., Jiang et al., 2011).

The late Ediacaran Dengying Formation in Yangtze Gorges area can be subdivided into three members from the base to top: 1) Hamajing Member with ca. 21-m-thick intraclastic and oolitic dolomitic grainstone; 2) Shibantan Member with ca. 50-m-thick dark grey laminated micritic limestone and cherty laminae in the upper part; 3) Baimatuo Member with ca. 40-mthick micritic and sparitic dolostones. The basal Dengying Formation contains a volcanic ash layer that has been dated at $551.1 \mathrm{Ma} \pm 0.7 \mathrm{Ma}$ with a ID-TIMS zircon U-Pb method (Condon et al., 2005). In the Dengying Formation, deep-water deposition (after a large transgression) is indicated by cherty laminae in the upper part of the Shibantan member, and shallow water is indicated by birds-eye structures and boxwork in the Baimatuo Member. Overlying the Dengying Formation is the Yanjiahe Formation, which spans the Ediacaran-Cambrian boundary (Jiang et al., 2012 and references therein). The 54-m-thick Yanjiahe Formation is characterized by two sub-cycles of shoaling. The lower sub-cycle $(\sim 29 \mathrm{~m})$ consists of dark grey laminated dolostone with cherty dolostone, siliceous layers and black shale, and dolostone with cherty and phosphatic clasts. The upper sub-cycle $(\sim 25 \mathrm{~m})$ is characterized by black siliceous rock with laminated black shale, laminated limestone interbedded with black shale, and limestone with siliceous and phosphatic clasts. The Yanjiahe Formation yields early Cambrian small shelly fossils in the dolostone layer with phosphatic clasts (SS1) and upper phosphatic limestone (SS3) (Jiang et al., 2012). The Ediacaran-Cambrian boundary is suggested to be at the bottom of the lower dolostone layer where the small shelly fossil assemblage zone I first occurs (Chen, 1984). The Yanjiahe Formation is overlain by Shuijingtuo Formation with stratigraphic discontinuity between the two formations. The Shuijingtuo Formation can be correlated with the Niutitang Formation or Jiumenchong Formation in slope and basin area, South China, which is considered the base of Cambrian Stage 3 (Jiang et al., 2012). 


\subsection{Xiaotan section}

The Xiaotan section is located on the southern bank of the Jinsha River, NE Yunnan Province, which represents an inter shelf sedimentary environment (Li et al., 2013). The Xiaotan section comprises, from the oldest to youngest, late Ediacaran Dengying Formation (upper Donglongtan Member, Jiucheng Member and Baiyanshao Member), early Cambrian Zhujiaqing Formation (the Daibu Member, Zhongyicun Member, Dahai Member), Shiyantou Formation, Yu'anshan Formation, Hongjingshao Formation, and middle Cambrian Wulongqing Formation. In the Dengying Formation, the Donglongtan Member consists of laminated dolostone; and the Jiucheng Member consists of shale that has been eroded away by the river; and the Baiyanshao Member contains thickly bedded to massive dolostone with colors of grey to dark grey. In the Cambrian Zhujiaqing Formation, the Daibu Member consists of interbedded thin-intermediate, dark, dolomitic cherts and pale yellowish siliceous dolosutone, representing a transgressive system tract. The Zhongyicun Member, overlying the Daibu Member, is a phosphatic unit with grey, thick bed of laminated phosphorite. Small shelly fossil assemblage (SS1 and SS2) have been found in the Zhongyicun Member. At the correlative Meishuncun section, a SIMS U-Pb zircon age of $535.2 \pm 1.7 \mathrm{Ma}$ has been reported from the middle of the Zhongyicun Member (Zhu et al., 2009). The Dahai Member consists of pale grey, thickly bedded limestone and contains small shelly fossil assemblage (SS3). The Shiyantou Formation comprises grey to dark grey, bedded quartz siltstone in its lower part, representing a condensed deposition and dark grey to black shale with small shelly assemblage (SS4) in the upper part (Li and Xiao, 2014). A SHRIMP U-Pb zircon age of $526 \pm 1.1 \mathrm{Ma}$ was reported for the basal Shiyantou Formation in the correlative Meishucun section (Compston et al., 2008). The Yu'anshan Formation, which is characterized by the first appearance datum (FAD) of trilobites in the basal formation, consists of black shale in the lower part and carbonate-rich siltstone in the upper part. The Hongjingshao Formation comprises dark reddish thickly bedded sandstone. The Wulongqing Formation in the Xiaotan section comprises grey thickly bedded limestone, interbedded yellow silty mudstone, which is suggested to be deposited in the lower Cambrian Stage 4 (Zhu et al., 2010).

\section{DR4. Uranium mass balance model}

Under the steady-state of global uranium mass balance, $\delta^{238} U$ values of global seawater is determined by the fractions of uranium fluxes to sediments in the three redox settings: anoxic 
$\left(f_{A O x}\right)$, suboxic $\left(f_{S O x}\right)$, and oxic $\left(f_{O x}\right)$. If riverine uranium is considered as the main input of oceanic uranium reservoir, we have

$$
F_{\text {river }}=F_{A O X}+F_{S O x}+F_{O x}=F_{\text {Total }} ; \quad f_{A O X}+f_{S O x}+f_{O x}=1
$$

Isotope mass balance is described by:

$$
\delta_{\text {river }}=\delta_{\text {Aox }} \cdot f_{A O x}+\delta_{\text {SOx }} \cdot f_{\text {SOx }}+\delta_{O x} \cdot f_{O x}
$$

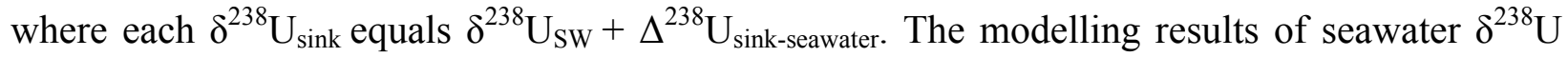
values as a function of $\mathrm{F}_{\mathrm{AOx}} / \mathrm{F}_{\text {Total }}$ and $\mathrm{F}_{\mathrm{Ox}} / \mathrm{F}_{\text {Total }}$ are shown in Fig. $2 \mathrm{~A}$ in the main text.

The uranium output rates $\left(\mathrm{F}_{\text {output }}\right.$ mol $\left.\mathrm{m}^{-2} \mathrm{yr}^{-1}\right)$ in various redox settings are assumed to be controlled by first-order kinetics with respect to the coeval uranium reservoir in the global ocean (R), that is, each $\mathrm{F}_{\text {output }}=\mathrm{F}_{\text {output } 0} \times \mathrm{R} / \mathrm{R}_{0}$ (subscript 0 represents the modern values). Replacing each $\mathrm{f}_{\text {output }}\left(=\mathrm{F}_{\text {output }} / \mathrm{F}_{\text {Total }}\right)$ in the isotope mass balance equation with:

$\frac{\left(F_{\text {output } 0} \cdot R / R_{0}\right) \cdot\left(A_{\text {Total }} \cdot f_{\text {output }}^{A}\right)}{F_{\text {Total }}}$

We can get the seawater $\delta^{238} U$ values as a function of areal proportions of anoxic condition and suboxic condition $\left(\mathrm{A}_{\mathrm{AOx}} / \mathrm{A}_{\text {Total }}\right.$ and $\mathrm{A}_{\mathrm{SOx}} / \mathrm{A}_{\text {Total }}$, respectively). The modelling results of seawater $\delta^{238} \mathrm{U}$ values as a function of $\mathrm{A}_{\mathrm{AOx}} / \mathrm{A}_{\text {Total }}$ and $\mathrm{A}_{\mathrm{SOX}} / \mathrm{A}_{\text {Total }}$ are shown in Fig. $2 \mathrm{~B}$ in the main text.

In modern suboxic settings, the $U$ isotope fractionation associated with suboxic sinks is less well constrained. The $+0.1 \%$ fractionation for suboxic sinks used in this study is based on a few measurements of Peru margin sediments (Weyer et al., 2008). In modern anoxic settings, the $\mathrm{U}$ isotope fractionation for anoxic sinks are relatively large and range from $+0.4 \%$ to $+0.85 \%$ o (Weyer et al., 2008; Andersen et al., 2014; Holmden et al., 2015; Stirling et al., 2015; Tissot and Dauphas, 2015; Rolison et al., 2017). $+0.7 \%$ is chosen as the average $U$ isotope fractionation between the anoxic sediment and seawater for our baseline model. For modern oxic settings, oxic $\mathrm{U}$ sinks include marine carbonates, metallic deposits (Fe-Mn crust), oceanic crust alteration, pelagic clay, and coastal retention. The average $U$ isotope fractionation from modern oxic sediments is $-0.043 \%$, modified after Wang et al. (2016), where $U$ isotope fractionation between seawater and marine carbonate is considered to be $0 \%$ (Anderson et al., 2017).

Baseline parameters used in the mass balance model are shown in Table DR2. Sensitivity analyses of changing $U$ isotope fractionation between seawater and anoxic/suboxic sediments are 
shown in Fig. DR4. Larger $U$ fractionation factors between seawater and anoxic/subxoic sediments result in even smaller areas of anoxic seafloor and greater predominance of oxic bottom waters for modern-like $\delta^{238} \mathrm{U}$ values. 
Table DR1. Geochemical data of the Gaojiaxi-Yanjiahe section and the Xiaotan section

\begin{tabular}{|c|c|c|c|c|c|c|c|c|c|c|c|c|}
\hline Sample & Formation & $\begin{array}{c}\text { Height } \\
\text { (m) }\end{array}$ & $\begin{array}{l}\delta^{13} \mathrm{C} \\
(\% \circ)\end{array}$ & $\begin{array}{c}\delta^{18} O \\
(\% 0)\end{array}$ & $\begin{array}{c}\text { Mg/Ca } \\
\text { (weight) }\end{array}$ & $\begin{array}{c}\text { U/Ca } \\
* 1000 \\
\end{array}$ & $\begin{array}{c}\mathrm{Sr} / \mathrm{Ca} \\
* 1000\end{array}$ & $\begin{array}{c}\delta^{238} U \\
(\% 0)\end{array}$ & ${ }^{234} \mathbf{U} /{ }^{238} \mathbf{U}$ & $\mathrm{Ce} / \mathrm{Ce} *$ & $\begin{array}{c}\text { Y/Ho } \\
\text { (weight) }\end{array}$ & $\begin{array}{c}\text { Th } \\
\text { (ppm) }\end{array}$ \\
\hline \multicolumn{13}{|c|}{ Gaojiaxi-Yanjiahe section } \\
\hline YJH-1 & Yanjiahe & 266.7 & -1.05 & -7.76 & 0.01 & 0.05 & 20.75 & -0.40 & 1.04 & 0.46 & 47.21 & 0.03 \\
\hline YJH-3 & Yanjiahe & 264.7 & -2.46 & -7.96 & 0.01 & 0.03 & 8.60 & -0.51 & 1.07 & 0.52 & 48.66 & 0.008 \\
\hline YJH-7 & Yanjiahe & 261.4 & 3.9 & -7.93 & 0.02 & 0.04 & 17.38 & -0.89 & 1.13 & 0.69 & 43.35 & 0.023 \\
\hline YJH-12 & Yanjiahe & 258.7 & 2.66 & -7.42 & 0.01 & 0.04 & 12.50 & -0.83 & 1.12 & 0.77 & 52.05 & 0.016 \\
\hline YJH-13 & Yanjiahe & 257.7 & -3.06 & -6.82 & 0.31 & 0.03 & 10.14 & -0.56 & 0.97 & 0.70 & 46.09 & 0.026 \\
\hline YJH-15 & Yanjiahe & 257.1 & -2.94 & -6.50 & 0.57 & 0.02 & 5.22 & -0.42 & 0.96 & 0.75 & 38.67 & 0.011 \\
\hline YJH-17 & Yanjiahe & 256.7 & -1.99 & -5.83 & 0.31 & 0.03 & 13.62 & -0.40 & 0.95 & 0.68 & 40.39 & 0.008 \\
\hline YJH-23 & Yanjiahe & 250.7 & -3.19 & -5.76 & 0.60 & 0.08 & 2.67 & -0.23 & 1.05 & 0.67 & 41.46 & 0.004 \\
\hline YJH-31 & Yanjiahe & 243.1 & 0.01 & -6.20 & 0.60 & 0.05 & 3.68 & -0.53 & 1.34 & 0.62 & 46.48 & 0 \\
\hline YJH-32 & Yanjiahe & 242.3 & 0.85 & -6.03 & 0.61 & 0.01 & 4.82 & -0.38 & 0.95 & 0.65 & 39.67 & 0.001 \\
\hline YJH-37 & Yanjiahe & 241 & 0.55 & -5.39 & 0.63 & 0.02 & 4.06 & -0.78 & 1.06 & 0.47 & 41.44 & 0.005 \\
\hline YJH-39 & Baimatuo Mb. & 240.6 & 0.46 & -5.65 & 0.62 & 0.01 & 2.33 & -0.46 & 1.07 & 0.29 & 37.94 & 0.007 \\
\hline YJH-43 & Baimatuo Mb. & 234.5 & 2.37 & -5.39 & 0.62 & 0.05 & 3.01 & -0.89 & 0.98 & 0.40 & 43.7 & 0.004 \\
\hline YJH-45 & Baimatuo Mb. & 231.1 & 2.94 & -5.63 & 0.62 & 0.05 & 3.23 & -0.98 & 1.03 & 0.33 & 42.86 & 0 \\
\hline YJH-47 & Baimatuo Mb. & 227.9 & 2.87 & -5.95 & 0.64 & 0.04 & 2.30 & -1.17 & 1.04 & 0.52 & 45.4 & 0.008 \\
\hline YJH-49 & Baimatuo Mb. & 223.9 & 2.81 & -5.00 & 0.62 & 0.03 & 1.86 & -1.18 & 1.06 & 0.55 & 50 & 0 \\
\hline YJH-51 & Baimatuo Mb. & 214.9 & 2.9 & -5.00 & 0.63 & 0.03 & 2.16 & -1.19 & 1.06 & 0.47 & 40.94 & 0.015 \\
\hline YJH-53 & Baimatuo Mb. & 210.9 & 2.92 & -5.85 & 0.64 & 0.03 & 2.22 & -1.01 & 1.02 & 0.53 & 43.25 & 0 \\
\hline YJH-55 & Baimatuo Mb. & 207.4 & 2.91 & -5.13 & 0.63 & 0.03 & 2.01 & -1.20 & 1.04 & 0.54 & 43.05 & 0 \\
\hline YJH-57 & Baimatuo Mb. & 204.4 & 2.82 & -5.20 & 0.64 & 0.03 & 2.04 & -1.10 & 1.03 & 0.55 & 43.86 & 0 \\
\hline YJH-59 & Baimatuo Mb. & 201.9 & 3.08 & -5.18 & 0.63 & 0.05 & 2.39 & -0.72 & 1.02 & 0.58 & 41.16 & 0 \\
\hline YJH-61 & Baimatuo Mb. & 199.9 & 2.31 & -5.08 & 0.63 & 0.04 & 1.97 & -1.09 & 1.02 & 0.54 & 36.23 & 0 \\
\hline \multicolumn{13}{|c|}{ Xiaotan section } \\
\hline $\begin{array}{c}\text { WLQ- } \\
717\end{array}$ & Wulongqing & 717 & -1.52 & -8.85 & 0.02 & 0.01 & 4.85 & -0.41 & 1.01 & 0.61 & 46.18 & 0.34 \\
\hline $\begin{array}{c}\text { WLQ- } \\
707\end{array}$ & Wulongqing & 707 & -2.61 & -7.17 & 0.06 & 0.02 & 3.76 & -0.39 & 1.04 & 0.52 & 37.09 & 0.16 \\
\hline $\begin{array}{c}\text { WLQ- } \\
674\end{array}$ & Wulongqing & 674 & -1.31 & -10.12 & 0.05 & 0.01 & 13.31 & -0.45 & 0.99 & 0.56 & 38.93 & 0.61 \\
\hline $\begin{array}{c}\text { WLQ- } \\
669\end{array}$ & Wulongqing & 669 & -1.39 & -9.43 & 0.12 & 0.01 & 11.25 & -0.43 & 1.04 & 0.43 & 40.49 & 0.43 \\
\hline $\begin{array}{c}\text { WLQ- } \\
664\end{array}$ & Wulongqing & 664 & -2.14 & -8.23 & 0.07 & 0.03 & 9.85 & -0.47 & 1.03 & 0.52 & 46.53 & 0.28 \\
\hline $\begin{array}{l}\text { WLQ- } \\
648.9\end{array}$ & Wulongqing & 648.9 & -2.01 & -7.12 & 0.01 & 0.01 & 5.99 & -0.38 & 1.05 & 0.51 & 47.51 & 0.47 \\
\hline $\begin{array}{c}\text { WLQ- } \\
642\end{array}$ & Wulongqing & 642 & -1.06 & -7.45 & 0.01 & 0.01 & 8.18 & -0.45 & 1.11 & 0.42 & 38.88 & 0.52 \\
\hline $\begin{array}{l}\text { WLQ- } \\
637.5\end{array}$ & Wulongqing & 637.5 & -2.6 & -10.8 & 0.02 & 0.02 & 8.28 & -0.42 & 0.98 & 0.49 & 36.09 & 0.43 \\
\hline $\begin{array}{c}\text { WLQ- } \\
631\end{array}$ & Wulongqing & 631 & -1.13 & -9.12 & 0.04 & 0.03 & 6.87 & -0.41 & 0.98 & 0.41 & 37.74 & 0.45 \\
\hline
\end{tabular}




\begin{tabular}{|c|c|c|c|c|c|c|c|c|c|c|c|c|}
\hline $\begin{array}{l}\text { WLQ- } \\
618.9\end{array}$ & Wulongqing & 618.9 & -0.99 & -7.67 & 0.02 & 0.02 & 7.45 & -0.43 & 1.01 & 0.47 & 38.44 & 0.28 \\
\hline XT-101 & Dahai & 278.9 & -4.11 & -14.96 & 0.01 & 0.02 & 17.71 & -0.38 & 1.13 & 0.47 & 47.23 & 0.35 \\
\hline XT-103 & Dahai & 277.9 & -0.94 & -14.49 & 0.02 & 0.03 & 37.88 & -0.64 & 1.46 & 0.59 & 46.64 & 0.13 \\
\hline XT-106 & Dahai & 275.5 & -0.22 & -15.11 & 0.01 & 0.04 & 47.18 & -0.72 & 1.65 & 0.42 & 42.15 & 0.21 \\
\hline XT-110 & Dahai & 272.5 & 4.16 & -11.89 & 0.00 & 0.03 & 14.99 & -0.74 & 1.27 & 0.56 & 43.95 & 0.45 \\
\hline XT-111 & Dahai & 270.5 & 5.52 & -11.18 & 0.00 & 0.06 & 55.51 & -0.76 & 1.38 & 0.48 & 46.12 & 0.08 \\
\hline XT-117 & Dahai & 261.9 & 7.22 & -11.46 & 0.01 & 0.08 & 112.59 & -0.68 & 1.65 & 0.62 & 48.16 & 0.07 \\
\hline XT-120 & Dahai & 258.2 & 7.06 & -10.99 & 0.01 & 0.12 & 134.3 & -0.83 & 1.17 & 0.48 & 46.05 & 0.12 \\
\hline XT-125 & Dahai & 251.7 & 6.66 & -12.08 & 0.01 & 0.09 & 110.48 & -0.99 & 0.97 & 0.57 & 45.65 & 0.23 \\
\hline XT-130 & Dahai & 244.3 & 6.71 & -11.28 & 0.01 & 0.12 & 117.27 & -0.85 & 1.18 & 0.55 & 44.76 & 0.36 \\
\hline XT-135 & Dahai & 237.5 & 7.15 & -12.17 & 0.01 & 0.08 & 51.59 & -0.77 & 1.29 & 0.4 & 44.87 & 0.11 \\
\hline XT-138 & Dahai & 233.3 & 7.11 & -10.90 & 0.01 & 0.09 & 78.33 & -0.66 & 0.99 & 0.44 & 45.12 & 0.08 \\
\hline XT-140 & Dahai & 230.8 & 5.96 & -12.07 & 0.01 & 0.14 & 65.16 & -0.60 & 1.02 & 0.4 & 44.65 & 0.07 \\
\hline XT-145 & Dahai & 222.6 & 6.53 & -11.36 & 0.00 & 0.05 & 47.72 & -0.38 & 0.99 & 0.49 & 46.12 & 0.35 \\
\hline XT-148 & Dahai & 218.1 & 5.83 & -11.84 & 0.00 & 0.07 & 80.82 & -0.50 & 1.04 & 0.41 & 48.14 & 0.15 \\
\hline XT-153 & Dahai & 213.1 & 1.05 & -11.82 & 0.00 & 0.1 & 108.6 & -0.59 & 1.10 & 0.45 & 42.15 & 0.23 \\
\hline XT-158 & Zhongyicun & 209.2 & -1.36 & -10.70 & 0.01 & 0.07 & 65.79 & -0.55 & 1.15 & 0.42 & 43.65 & 0.45 \\
\hline XT-165 & Zhongyicun & 204 & -6.44 & -13.10 & 0.01 & 0.08 & 79.03 & -0.48 & 1.27 & 0.41 & 44.86 & 0.52 \\
\hline XT-178 & Zhongyicun & 193.5 & -2.97 & -9.95 & 0.01 & 0.07 & 80.39 & -0.50 & 1.30 & 0.32 & 43.65 & 0.02 \\
\hline XT-190 & Zhongyicun & 179.9 & -5.29 & -9.02 & 0.02 & 0.04 & 36.41 & -0.51 & 1.16 & 0.44 & 43.25 & 0.34 \\
\hline XT-201 & Zhongyicun & 168.7 & -4.38 & -10.01 & 0.11 & 0.06 & 57.15 & -0.47 & 1.28 & 0.55 & 46.14 & 0.56 \\
\hline XT-204 & Zhongyicun & 168.1 & -4.32 & -8.50 & 0.34 & 0.02 & 9.27 & -0.64 & 1.44 & 0.54 & 43.63 & 0.60 \\
\hline XT-212 & Zhongyicun & 162.5 & -4.84 & -10.23 & 0.13 & 0.03 & 15.09 & -0.24 & 1.25 & 0.37 & 46.34 & 0.54 \\
\hline XT-224 & Zhongyicun & 152.5 & -3.41 & -9.38 & 0.39 & 0.01 & 3.49 & -0.47 & 1.12 & 0.46 & 45.81 & 0.32 \\
\hline XT-238 & Daibu & 106.9 & -5.51 & -9.07 & 0.29 & 0.05 & 3.22 & -0.46 & 1.13 & 0.52 & 44.32 & 0.15 \\
\hline XT-241 & Daibu & 103.6 & -4.86 & -8.40 & 0.72 & 0.01 & 2.83 & -0.42 & 1.21 & 0.37 & 41.26 & 0.26 \\
\hline XT-246 & Daibu & 97.9 & -7.27 & -7.62 & 0.48 & 0.02 & 3.16 & -0.49 & 1.21 & 0.37 & 45.65 & 0.34 \\
\hline XT-253 & Daibu & 87.7 & -13.51 & -17.97 & 0.42 & 0.03 & 3.46 & -0.51 & 1.17 & 0.39 & 45.34 & 0.19 \\
\hline XT-259 & Daibu & 80.6 & -12.18 & -7.09 & 0.26 & 0.04 & 2.82 & -0.28 & 1.11 & 0.43 & 45.98 & 0.10 \\
\hline XT-270 & Baiyanshao & 70 & 0.19 & -7.75 & 0.34 & 0.04 & 2.62 & -0.30 & 1.08 & 0.51 & 44.12 & 0.01 \\
\hline XT-285 & Baiyanshao & 48.4 & 1.35 & -6.49 & 0.36 & 0.04 & 2.97 & -0.68 & 1.30 & 0.49 & 42.67 & 0.01 \\
\hline XT-297 & Baiyanshao & 27.3 & 2.26 & -5.73 & 0.27 & 0.02 & 2.46 & -0.71 & 1.07 & 0.52 & 47.21 & 0.01 \\
\hline XT-304 & Baiyanshao & 15 & 2.50 & -3.65 & 0.36 & 0.05 & 1.57 & -0.97 & 1.25 & 0.57 & 46.86 & 0.01 \\
\hline XT-312 & Baiyanshao & 0 & 2.98 & -3.52 & 0.39 & 0.03 & 3.65 & -1.07 & 1.24 & 0.66 & 44.15 & 0.02 \\
\hline XT-314 & Donglongtan & -22 & 1.30 & -6.83 & 0.29 & 0.02 & 2.41 & -1.14 & 1.38 & 0.72 & 42.17 & 0.01 \\
\hline XT-316 & Donglongtan & -26 & 0.82 & -7.43 & 0.27 & 0.03 & 1.69 & -1.07 & 1.28 & 0.7 & 41.56 & 0.03 \\
\hline XT-318 & Donglongtan & -30 & 0.96 & -7.06 & 0.26 & 0.03 & 2.90 & -0.87 & 1.39 & 0.82 & 44.61 & 0.01 \\
\hline XT-321 & Donglongtan & -36 & 0.90 & -7.08 & 0.38 & 0.01 & 2.46 & -1.06 & 1.09 & 0.72 & 45.19 & 0.01 \\
\hline
\end{tabular}


Table DR2. Parameters of the uranium isotope mass balance model

\begin{tabular}{|c|c|c|c|c|}
\hline Parameter & Description & Value & Unit & Reference \\
\hline $\mathrm{F}_{\text {river }}$ & riverine $\mathrm{U}$ flux to ocean & $4.2 \times 10^{7}$ & $\mathrm{~mol} / \mathrm{yr}$ & $\begin{array}{c}\text { Anderson et al. } 2017 \text { for a } \\
\text { review }\end{array}$ \\
\hline $\mathrm{F}_{\mathrm{Ox} 0}$ & Modern output flux to oxic U sink & $2.23 \times 10^{7}$ & $\mathrm{~mol} / \mathrm{yr}$ & $\begin{array}{c}\text { Anderson et al. } 2017 \text { for a } \\
\text { review }\end{array}$ \\
\hline $\mathrm{F}_{\mathrm{SOx} 0}$ & Modern output flux to subxoic $\mathrm{U}$ sink & $1.53 \times 10^{7}$ & $\mathrm{~mol} / \mathrm{yr}$ & $\begin{array}{c}\text { Anderson et al. } 2017 \text { for a } \\
\text { review }\end{array}$ \\
\hline $\mathrm{F}_{\mathrm{AOx} 0}$ & Modern out flux to anoxic U sink & $4.45 \times 10^{6}$ & $\mathrm{~mol} / \mathrm{yr}$ & $\begin{array}{l}\text { Anderson et al. } 2017 \text { for a } \\
\text { review }\end{array}$ \\
\hline$\delta_{\text {river }}$ & riverine $\delta^{238} \mathrm{U}$ value & -0.29 & $\%$ & $\begin{array}{l}\text { Anderson et al. } 2017 \text { for a } \\
\text { review } \\
\end{array}$ \\
\hline$\delta_{\text {seawater } 0}$ & modern seawater $\delta^{238} U$ value & -0.4 & $\%$ & $\begin{array}{l}\text { Anderson et al. } 2017 \text { for a } \\
\text { review }\end{array}$ \\
\hline$\Delta_{\mathrm{Ox}}$ & $\begin{array}{c}\text { U fractionation between oxic sink and } \\
\text { seawater }\end{array}$ & -0.043 & $\%$ & revised from Wang et al. 2016 \\
\hline$\Delta_{\mathrm{SOx}}$ & $\begin{array}{l}\mathrm{U} \text { fractionation between suboxic sink } \\
\text { and seawater }\end{array}$ & 0.1 & $\%$ & Weyer et al. 2008 \\
\hline$\Delta_{\mathrm{AOx}}$ & $\begin{array}{l}\text { U fractionation between anoxic sink } \\
\text { and seawater }\end{array}$ & 0.7 & $\%$ & $\begin{array}{l}\text { revised from Anderson et al. } \\
\text { 2014; Holmden et al. 2015; } \\
\text { Rolison et al. } 2017\end{array}$ \\
\hline V & seawater volume & $1.37 \times 1021$ & $\mathrm{dm} 3$ & Hastings et al. 1996 \\
\hline A & Total seafloor area & $3.61 \times 1016$ & $\mathrm{dm} 2$ & Barnes and Cochran, 1990 \\
\hline $\mathrm{A}_{\mathrm{AOx}}$ & modern anoxic seafloor area & 0.35 & $\%$ & Veeh, 1968 \\
\hline $\mathrm{A}_{\mathrm{SOx}}$ & modern suboxic seafloor area & 6.00 & $\%$ & Dunk et al. 2002 \\
\hline $\mathrm{A}_{\mathrm{Ox}}$ & modern anoxic seafloor area & 93.65 & $\%$ & Balance \\
\hline$\tau$ & $\begin{array}{l}\text { residence time of uranium in the } \\
\text { ocean }\end{array}$ & 400 & kyr & Ku et al. 1977 \\
\hline
\end{tabular}




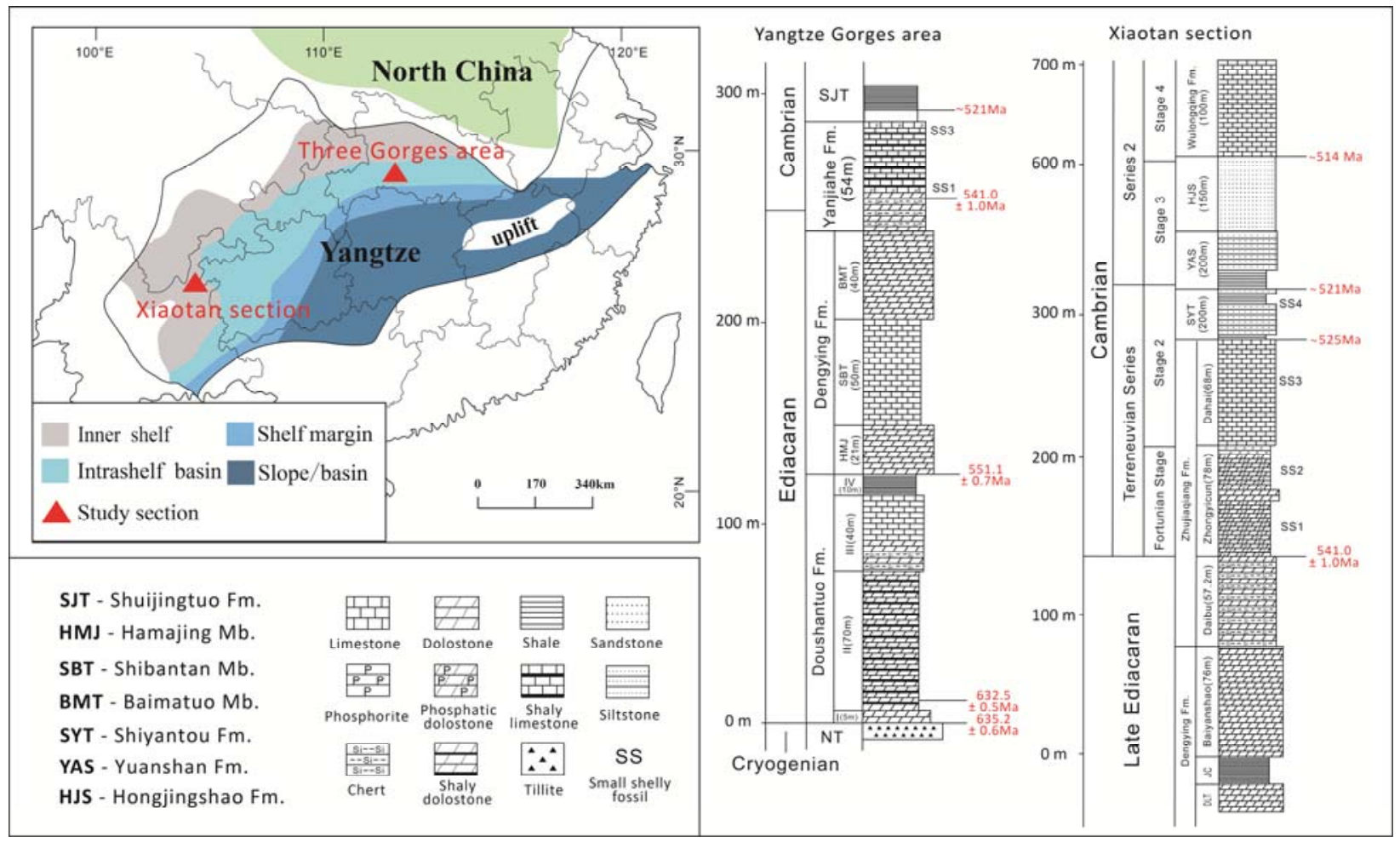

Fig DR1. Simplified geological map of the Yangtze block (after Jiang et al., 2012) and stratigraphic columns of the Yangtze Gorges area and Xiaotan section. The ages of every formation in the studied sections are modified from Ling et al. (2013); Li et al. (2013) and references therein. 

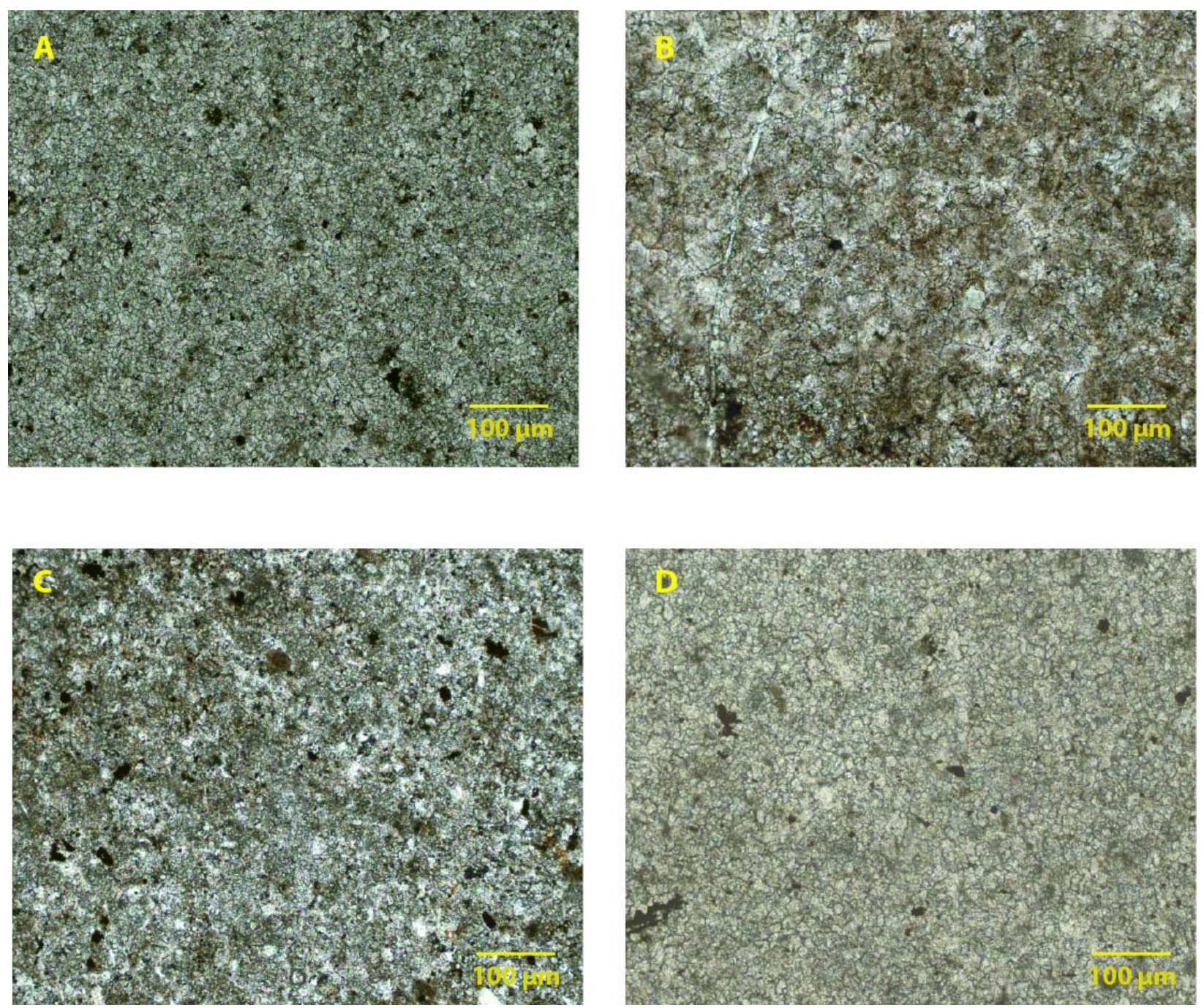

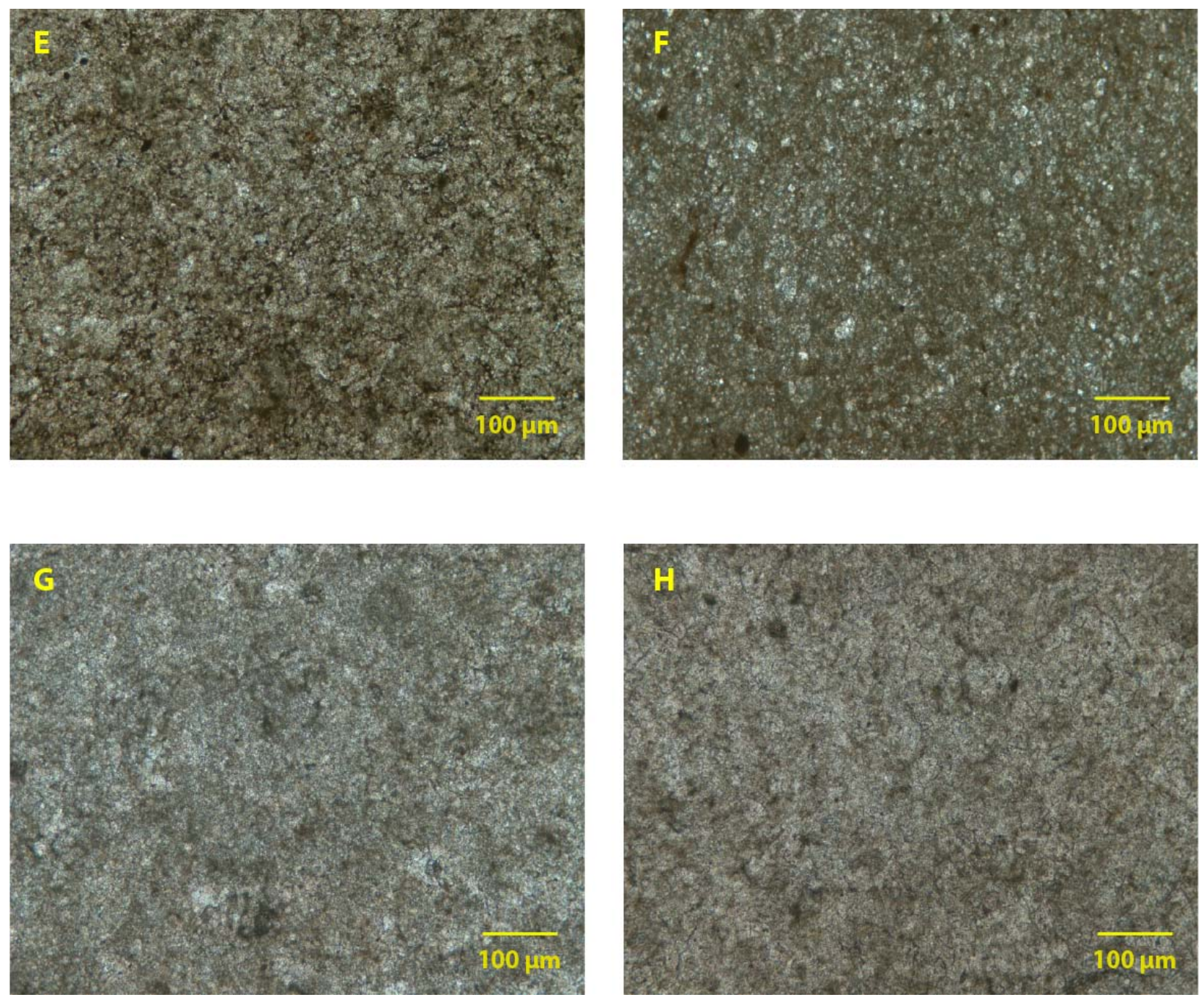

Fig DR2. Thin sections from studied carbonate samples under polarizing light microscope. (A) micrite or microsparite in the Dahai Mb., Xiaotan section; (B) dolomicrite interbedded in phosphorite in the Zhongyicun Mb., Xiaotan section; (C) coarsely grained cherty dolostone in the Daibu Mb., Xiaotan section; (D) dolosparite or dolomicrosparite in the Dengying Fm., Xiaotan section; (E) fine-grained micrite in the upper Yanjiahe Fm., Gaojiaxi-Yanjiahe section (F) microsparite in the upper Yanjiahe Fm., Gaojiaxi-Yanjiahe section; (G) dolomicrosparite in the lower Yanjiahe Fm., Gaojiaxi-Yanjiahe section. $(\mathrm{H})$ dolomicrite in the Dengying Fm. (Baimatuo Mb.), Gaojiaxi-Yanjiahe section. 

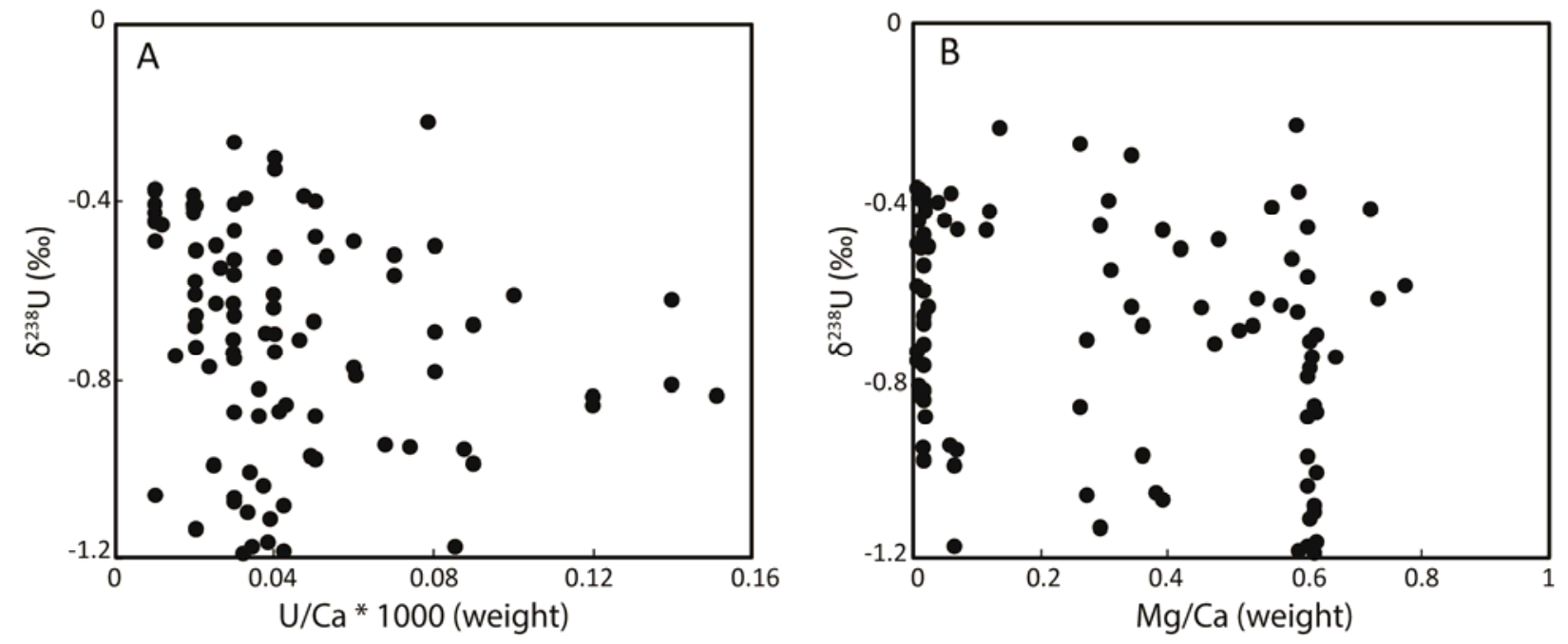

Fig DR3. Analyses of $\delta^{238} \mathrm{U}$ vs. U/Ca ratio (A) and $\mathrm{Mg} / \mathrm{Ca}$ ratio (B) as the tracers for effects of authigenic $U$ accumulation and dolomitization on $U$ isotopic composition in the carbonates. 

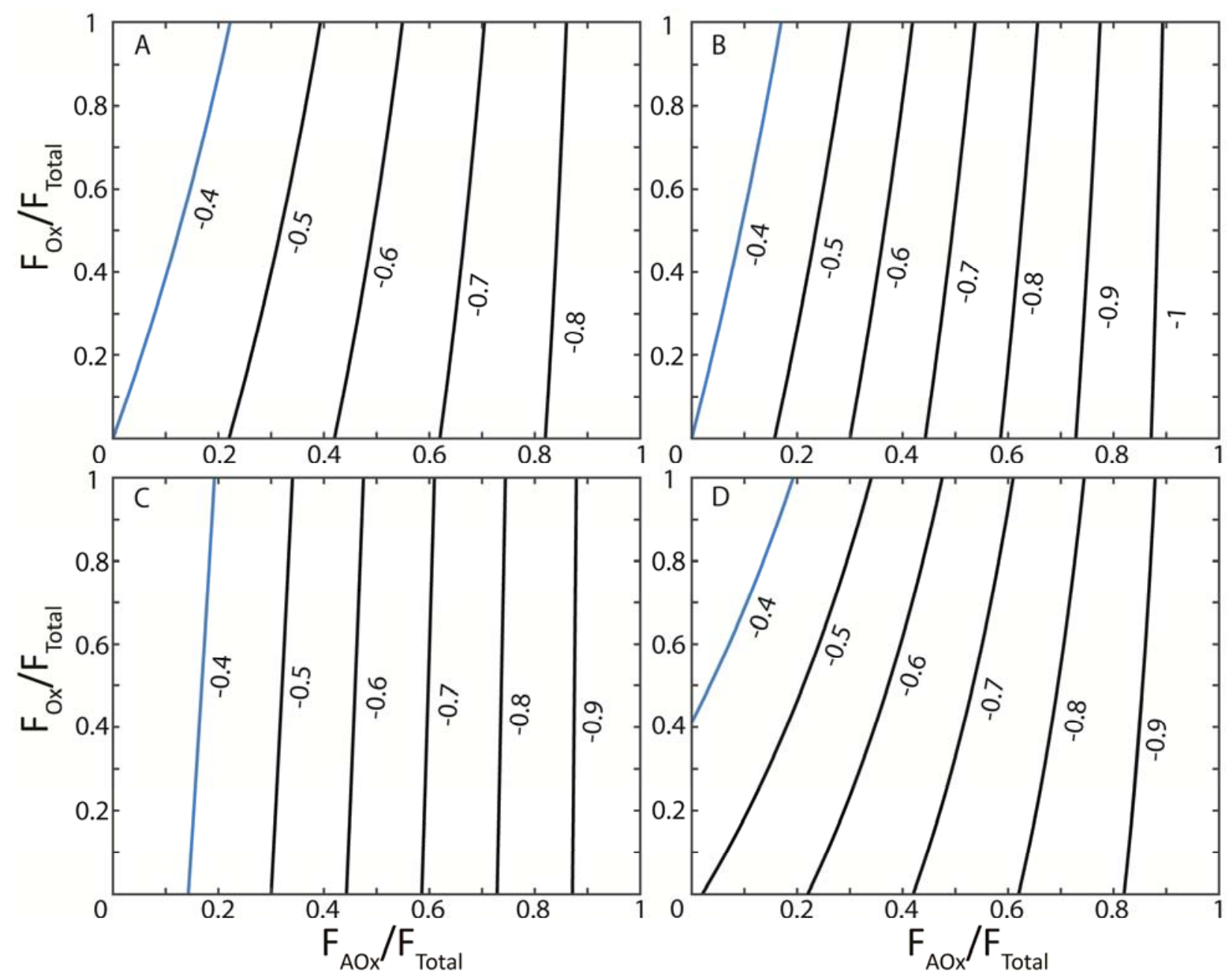

Fig DR4. Results of sensitivity analyses for $U$ isotope mass balance model. (A) $\Delta{ }^{238} \mathrm{U}_{\mathrm{AOx}-\mathrm{SW}}=$ $0.6 \%$; (B) $\Delta^{238} \mathrm{U}_{\mathrm{AOx}-\mathrm{SW}}=0.8 \%$; (C) $\Delta^{238} \mathrm{U}_{\mathrm{SOx}-\mathrm{SW}}=0 \%$; (D) $\Delta^{238} \mathrm{U}_{\mathrm{SOx}-\mathrm{SW}}=0.2 \%$. 


\section{References}

Andersen, M. B., Romaniello, S., Vance, D., Little, S. H., Herdman, R., and Lyons, T. W., 2014, A modern framework for the interpretation of ${ }^{238} \mathrm{U} /{ }^{235} \mathrm{U}$ in studies of ancient ocean redox: Earth and Planetary Science Letters, v. 400, p. 184-194.

Andersen, M. B., Stirling, C. H., and Weyer, S., 2017, Uranium Isotope Fractionation: Reviews in Mineralogy and Geochemistry, v. 82, p. 799-850.

Barnes, C. E., and Cochran, J. K., 1990, Uranium removal in oceanic sediments and the oceanic U balance: Earth and Planetary Science Letters, v. 97, p. 94-101.

Chen, P., 1984, Discovery of Lower Cambrian small shelly fossils from Jijiapo Yichang, west Hubei and its significance: Professional Papers of Stratigraphy and Palaeontology, v. 13, p. 49-66.

Cheng, H., et al., 2013, Improvements in ${ }^{230} \mathrm{Th}$ dating, ${ }^{230} \mathrm{Th}$ and ${ }^{234} \mathrm{U}$ half-life values, and U-Th isotopic measurements by multi-collector inductively coupled plasma mass spectrometry: Earth and Planetary Science Letters, v. 371-372, p. 82-91.

Compston, W., Zhang, Z., Cooper, J. A., Ma, G., and Jenkins, R. J. F., 2008, Further SHRIMP Geochronology on the early Cambrian of South China: American Journal of Science, v. 308, p. 399-420.

Condon, D., Zhu M., Bowring, S., Wang W., Yang, A., and Jin, Y., 2005, U-Pb Ages from the Neoproterozoic Doushantuo Formation, China: Science, v. 308, p. 95-98.

Dunk, R. M., Mills, R. A., and Jenkins, W. J., 2002, A reevaluation of the oceanic uranium budget for the Holocene: Chemical Geology, v. 190, p. 45-67.

Hastings, D. W., Emerson, S. R., and Mix, A. C., 1996, Vanadium in foraminiferal calcite as a tracer for changes in the areal extent of reducing sediments: Paleoceanography, v. 11(6), p. 665-678.

Holmden, C., Amini, M., and Francois, R., 2015, Uranium isotope fractionation in Saanich Inlet: A modern analog study of a paleoredox tracer: Geochimica et Cosmochimica Acta, v. 153 , p. 202-215.

Jiang, G., Shi, X., Zhang, S., Wang, Y., and Xiao, S., 2011, Stratigraphy and paleogeography of the Ediacaran Doushantuo Formation (ca. 635-551 Ma) in South China. Gondwana Research, v. 19(4), p. 831-849. 
Jiang, G., Wang, X., Shi, X., Xiao, S., Zhang, S., and Dong, J., 2012, The origin of decoupled carbonate and organic carbon isotope signatures in the early Cambrian (ca. 542-520 Ma) Yangtze platform: Earth and Planetary Science Letters, v. 317-318, p. 96-110.

Ku, T.-L., Knauss, K. G., and Mathieu, G. G., 1977, Uranium in open ocean: concentration and isotopic composition: Deep-Sea Research, v. 24, p. 1005-1017.

Lawrence, M. G., Greig, A., Collerson, K. D., and Kamber, B. S., 2006, Rare Earth Element and Yttrium Variability in South East Queensland Waterways: Aquatic Geochemistry, v. 12(1), p. 39-72.

Li, D., Ling, H.-F., Shields-Zhou, G. A., Chen, X., Cremonese, L., Och, L., Thirlwall, M., and Manning, C. J., 2013, Carbon and strontium isotope evolution of seawater across the Ediacaran-Cambrian transition: Evidence from the Xiaotan section, NE Yunnan, South China: Precambrian Research, v. 225, p. 128-147.

Li, G., and Xiao, S., 2004, Tannuolina and Micrina (Tannuolinidae) from the Lower Cambrian of eastern Yunnan, South China, and their scleritome reconstruction: Journal of paleontology, v. 78(5), p. 900-913.

Ling, H.-F., Chen, X., Li, D., Wang, D., Shields-Zhou, G. A., and Zhu, M., 2013, Cerium anomaly variations in Ediacaran-earliest Cambrian carbonates from the Yangtze Gorges area, South China: Implications for oxygenation of coeval shallow seawater: Precambrian Research, v. 225, p. 110-127.

McFadden, K. A., Huang, J., Chu, X., Jiang, G., Kaufman, A. J., Zhou, C., Yuan, X., and Xiao, S., 2008, Pulsed oxidation and biological evolution in the Ediacaran Doushantuo Formation: Proceedings of National Academy Sciences USA, v. 105(9), p. 3197-3202.

McLennan, S.M., 1989, Rare-earth elements in sedimentary-rocks - influence of provenance and sedimentary processes: Review in Mineralogy, v. 21, p. 169-200.

Rolison, J. M., Stirling, C. H., Middag, R., and Rijkenberg, M. J. A., 2017, Uranium stable isotope fractionation in the Black Sea: Modern calibration of the ${ }^{238} \mathrm{U} /{ }^{235} \mathrm{U}$ paleo-redox proxy: Geochimica et Cosmochimica Acta, v. 203, p. 69-88.

Stirling, C. H., Andersen, M. B., Warthmann, R., and Halliday, A. N., 2015, Isotope fractionation of ${ }^{238} \mathrm{U}$ and ${ }^{235} \mathrm{U}$ during biologically-mediated uranium reduction: Geochimica et Cosmochimica Acta, v. 163, p. 200-218. 
Tissot, F. L. H., and Dauphas, N., 2015, Uranium isotopic compositions of the crust and ocean: Age corrections, $\mathrm{U}$ budget and global extent of modern anoxia: Geochimica et Cosmochimica Acta, v. 167, p. 113-143.

Villa, I. M., Bonardi, M. L., De Bièvre, P., Holden, N. E., and Renne, P. R., 2016, IUPAC-IUGS status report on the half-lives of ${ }^{238} \mathrm{U},{ }^{235} \mathrm{U}$ and ${ }^{234} \mathrm{U}$ : Geochimica et Cosmochimica Acta, V. 172 , p. 387-392.

Wang, X., Planavsky, N.J., Reinhard, C. T., Hein, J. R., and Johnson, T. M., 2016. A Cenozoic seawater redox record derived from ${ }^{238} \mathrm{U} /{ }^{235} \mathrm{U}$ in ferromanganese crusts: American Journal of Science, v. 316, p. 64-83.

Weyer, S., Anbar, A. D., Gerdes, A., Gordon, G. W., Algeo, T. J., and Boyle, E. A., 2008, Natural fractionation of ${ }^{238} \mathrm{U} /{ }^{235} \mathrm{U}$ : Geochimica et Cosmochimica Acta, v. 72(2), p. 345359.

Yin, L. M., Zhu, M.Y., Knoll, A.H., Yuan, X.L., Zhang, J.M., and Hu, J., 2007, Doushantuo embryos preserved inside diapause egg cysts: Nature, v. 446, p. 661-663.

Zhu, M., 2010, The origin and Cambrian explosion of animals: Fossil evidence from China: Acta Palaeontologica Sinica, v. 49, p. 269-287 (in Chinese with English abstract).

Zhu, R., Li, X., Hou, X., Pan, Y., Wang, F., Deng, C., and He, H., 2009, SIMS U-Pb zircon age of a tuff layer in the Meishucun section, Yunnan, southwest China: Constraint on the age of the Precambrian-Cambrian boundary: Science in China Series D: Earth Sciences, v. 52(9), p. 1385-1392. 